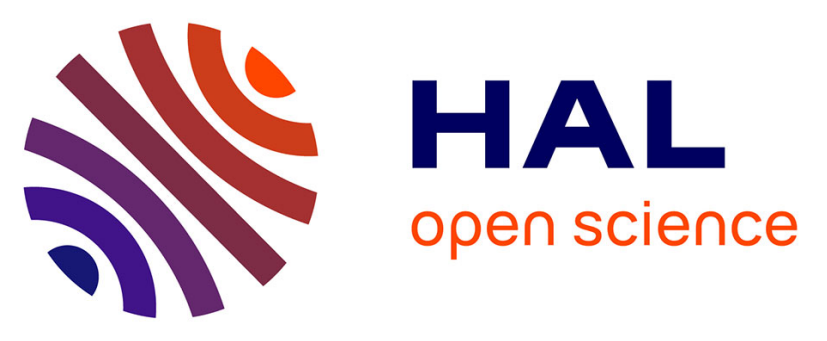

\title{
Chronique: Prise d'habit de la 138è Promotion de l'Ecole Forestière - La Compagnie Nationale des Experts Forestiers devient la Compagnie Nationale des Ingénieurs Experts et Techniciens de la Forêt Privée - La Chine continentale, marché possible pour le bois à pâte - Eau et air dans les plans d'aménagement régionaux - Les poteaux en bois pour lignes de distribution d'énergie électrique \\ - [.]revue Forestière Française, Rédaction
}

To cite this version:

- [.]revue Forestière Française, Rédaction. Chronique: Prise d'habit de la 138è Promotion de l'Ecole Forestière - La Compagnie Nationale des Experts Forestiers devient la Compagnie Nationale des Ingénieurs Experts et Techniciens de la Forêt Privée - La Chine continentale, marché possible pour le bois à pâte - Eau et air dans les plans d'aménagement régionaux - Les poteaux en bois pour lignes de distribution d'énergie électrique. 1965, pp.141. 10.4267/2042/24716 . hal-03535499

\author{
HAL Id: hal-03535499 \\ https://hal.science/hal-03535499
}

Submitted on 19 Jan 2022

HAL is a multi-disciplinary open access archive for the deposit and dissemination of scientific research documents, whether they are published or not. The documents may come from teaching and research institutions in France or abroad, or from public or private research centers.
L'archive ouverte pluridisciplinaire HAL, est destinée au dépôt et à la diffusion de documents scientifiques de niveau recherche, publiés ou non, émanant des établissements d'enseignement et de recherche français ou étrangers, des laboratoires publics ou privés. 


\section{CHRONIQUE}

\section{5 et 6 décembre 1964}

\section{Prise d'Habit de la 138 Promotion de l'Ecole Forestière}

Les Elèves de la $138^{\circ}$ Promotion de 1'Ecole Forestière ont pris 1'Habit le 5 décembre. Ce grand jour était attendu avec une impatience extrême, d'abord évidemment par les marauds eux-mêmes, ensuite par tous leurs anciens, heureux de voir le Corps forestier prouver de façon éclatante sa vitalité en mettant au monde une nombreuse nichée de « Jeunes Camarades 》. Il était attendu aussi par toute la ville de Nancy où l'Ecole tient la place que l'on sait. D'aucuns ont même prétendu que beaucoup de bonnes familles voyaient d'un œil point trop défavorable la sortie sur le marché d'un lot important de brillants uniformes tout neufs.

L'attente de tous allait être récompensée.

Au terme de plusieurs mois de préparation, après le défilé du jeudi 3 où un cortège d'élèves vêtus d'uniformes anciens avait annoncé l'événement à travers la ville parmi les fanfares des trompes de chasse de l'Ecole, vint le soir du Bal. Le hall de l'Hôtel de Ville était tout empli des animaux les plus féroces, aussi naturalisés que possible, qui marchaient, couraient, s'entr'égorgeaient au milieu des arbres d'une forêt assez sauvage toute frémissante de l'écho des fanfares que sonnait avec maîtrise le Débûché de Nancy. Ce décor assez abrupt, où près de sept cents personnes allaient passer, était parcouru sans cesse du bruissement des longues robes, de l'éclat des uniformes, et de la stricte élégance des habits.

Après le traditionnel vin d'honneur, auquel on remarquait particulièrement la présence de MM. les Ingénieurs Généraux Toussaint, Castagnou, Badré, LACHAUSSÉE et Viney, et l'entrée solennelle des jeunes forestiers sous la voûte d'acier qu'avaient formée leurs anciens, après que les honneurs eussent été rendus - et sonnés - aux personnalités, le Bal fut ouvert par une valse endiablée, interrompu pendant quelque temps par un intermède de qualité emprunté au spectacle du Cabaret «Echelle de Jacob » qui n'en brisa pas le rythme, et dura jusqu'à l'aube.

Les cérémonies du lendemain dimanche commencèrent par un hommage aux forestiers morts pour la France. Après l'Appel, qui eu lieu comme d'habitude dans la cour de 1'Ecole, une Messe de Saint-Hubert fut célébrée à leur mémoire par le R.P. LAURENCEAU O.P., aumônier des' élèves, devant l'assistance nombreuse qui emplissait la Cathédrale de Nancy. Le Débûché de Nancy fit, là encore, preuve de sa virtuosité, et cette messe fut en tout point admirable.

A l'apéritif qui la suivait, et qui réunissait autour des élèves leur famille, leurs anciens, et leurs amis, tous, et particulièrement la $138^{\circ}$ Promotion, eurent la joie de voir M: l'Ingénieur Général CASTAGnou remettre à M. Dolmain, Surveillant Général de $1^{\text {re }}$ Année, la croix de Chevalier du Mérite Agricole, et prononcer à cette occasion quelques mots dont chacun apprécia la fine érudition et l'à-propos. M. l'Ingénieur Général ViNEY fit avec gentillesse le discours qu'il fallait.

Puis vinrent les cérémonies du Baptême, dont je ne parlerai pas, puisque les Forestiers les ont connues et que les autres n'ont pas à les connaître. Paix aux mânes du. Premier Conservateur de France! 
Enfin se déroula le traditionnel banquet, auquel le Château de Lunéville prêtait un cadre somptueux. Les Missaires de trois promotions s'y distinguèrent et $\mathrm{y}$ rivalisèrent d'esprit. Le Débûché de Nancy envoya le bouquet du feu d'artifice de sonneries dont nous avions été éblouis pendant ces deux jours, M. le Conservateur LE PONT, parrain de la $138^{\circ}$ Promotion, se lança dans des variations brillantes, et $M$. l'Ingénieur Général Toussaint prononça le discours qu'il a coutume de prononcer en pareil cas. Tout cela devant près de cent personnes, Forestiers français et représentants des trois facultés forestières d'Allemagne.

Cette Prise d'Habit, que nous avions préparée dans l'inconnu, puisque l'an passé le Bal n'avait pu avoir lieu, que pour la première fois la Messe était célébrée à la Cathédrale et le banquet fait à Lunéville, que le Spectacle de variétés était une innovation, et qu'enfin les Elèves avaient voulu marquer cette date en distribuant une plaquette de qualité sur quelques-uns des aspects du métier de Forestier, a été un succès complet. Succès matériel, certes, grâce à l'effort de tous, mais aussi fête de famille forestière. En effet, les Elèves de la $138^{\mathrm{e}}$ Promotion n'étaient pas seuls à prendre l'Habit: M. le Directeur VINEY portait pour la première fois ces étoiles qu'il a si bien méritées, mais dont il nous a fait l'honneur de dire qu'elles étaient un peu celles de l'Ecole, et dont nous sommes si fiers. Puisse la Prise d'Habit de l'an prochain dépasser encore en splendeur, mais surtout en sympathie celle de cette année. Quelle que soit l'évolution que subisse notre métier, les traditions ne nous doivent pas être un poids. Elles sont une grande richesse. Souhaitons qu'elles ne soient pas anéanties sans discernement et que l'Ecole Forestière voie encore beaucoup de Prises d'Habit.

François Letourneux.

\section{La Compagnie Nationale des Experts Forestiers devient la Compagnie Nationale des Ingénieurs, Experts et Techniciens de la Forêt Privée}

La Compagnie Nationale des Experts Forestiers a tenu son Assemblée Générale annuelle le 30 octobre 1964, au Pavillon Dauphine à Paris.

Cette Assemblée Générale revêtait une importance toute particulière puisque, entre autres questions, son ordre du jour comprenait des propositions apportant de profondes modifications aux Statuts de la Compagnie.

- L'évolution récente de la structure et de l'organisation de la forêt privée devait entraîner également une évolution de la Compagnie Nationale des Experts Forestiers. Déjà, il y a deux ans, la création d'une Commission Technique avait montré son désir d'aller de l'avant. Son importante étude de «L'Aménagement des Forêts Privées 》 n'est ignorée de personne.

- Mais il fallait faire plus et ouvrir largement les portes à tous ceux qui, Ingénieurs, Experts ou Techniciens, travaillent dans un même idéal à l'amélioration et à la prospérité de la forêt privée.

C'est dans cet esprit que le Comité Directeur de la Compagnie s'était imposé depuis plusieurs mois la tâche de préparer une réforme des statuts.

C'est à une très large majorité que l'Assemblée Générale a approuvé cette réforme, manifestant ainsi sa volonté d'action.

La Compagnie Nationale des Experts Forestiers devient donc la:

$$
\begin{aligned}
& \text { COMPAGNIE NATIONALE DES INGENIEURS, } \\
& \text { EXPERTS ET TECHNICIENS DE LA FORET PRIVEE }
\end{aligned}
$$


C'est par acclamations que M. le Président ChaIR, qui tout au long d'un mandat de huit années a assumé avec une rare distinction cette lourde charge, a été nommé Président d'Honneur.

La Compagnie Nationale des Ingénieurs, Experts et Techniciens de la Forêt Privée a immédiatement procédé à l'élection de son Comité-Directeur, qui a été ainsi composé :

MM: Airy R., Barthelemy L., Bartmann, Camus, Chavet, Collardet, Dufay, Duret, Durieux, Dutilloy, de Grandcourt, Jousset, Juvanon du Vachat, Lanternier, Michaut, de La Potterie, Rothe.

Le Comité Directeur, réuni le 4 novembre 1964, a lui-même aussi constitué son Bureau:

Président: M. de Grandcourt.

Vice-Présidents: M: Collardet, M. Rothe.

Secrétaire Général: M. Bartmann.

Trésorier: M. Juvanon du Vachat.

\section{La Chine continentale marché possible pour le bois à pâte}

Rome, 3 novembre. - L'augmentation de la demande de bois en Chine continentale permet de penser que ce pays deviendra un des principaux acheteurs de produits forestiers, et notamment de bois à pâte et de pâte à papier. Ceux-ci ne peuvent provenir que de réserves de conifères se trouvant sur les côtes de l'Océan Pacifique dans le Nord de l'Amérique et le Nord-Est de l'U.R.S.S.

Cette éventualité a été évoquée lors de la réunion de la Commission des forêts Asie/Pacifique de la FAO (Organisation des Nations Unies pour l'alimentation et l'agriculture) qui s'est tenue à Reterua (Nouvelle-Zélande) du 22 septembre au 2 octobre 1964. La question a été traitée dans une étude sur la forêt en Chine continentale, rédigée par M. S.D. Richardson, directeur chargé de la recherche du Service néo-zélandais des forêts, étude qui a été soumise aux participants.

M. Richardson avait effectué un voyage en Chine en mai et juin 1963, au cours duquel il avait visité de nombreuses entreprises forestières et centres de recherches dans plusieurs provinces.

Selon l'auteur de l'étude, les besoins industriels en bois de la Chine continentale s'élèveront à 118,5 millions de mètres cubes en 1972 , alors que la production prévue n'atteindra que 80 millions. Le déficit sera donc de 38,5 millions et s'élèvera au cours des années suivantes jusqu'à 45 millions environ en 1977.

La Chine a surtout besoin de grosses grumes que l'Asie du Sud-Est possède en quantité et qu'elle peut exporter. Le bois et la pâte à papier que l'on trouve sur les côtes Pacifique de l'Amérique du Nord et de l'URSS seront sans doute les produits dont la demande augmentera le plus.

Les terres à forêts couvrent 96 millions d'hectares en Chine continentale soit, pour l'ensemble de la région Asie-Pacifique, le cinquième des régions forestières accessibles. Selon le Plan national chinois pour l'agriculture de 1956 à 1968, 105 millions d'hectares seront reboisés pour renforcer les ressources forestières existantes. On pensait, en 1956, que ce reboisement suffirait pour 30 ans.

"Le travail de reboisement en Chine a été prodigieux, déclare M. Richardson dans son étude, mais la mauvaise qualité des sites de plantation choisis et le mauvais entretien ont annihilé ces efforts... Dans certaines communes, moins de 10 pour cent des plants ont survécu. Peut-être est-ce là qu'il 
faut trouver l'explication de la diminution de la production annuelle de bois. Elle est tombée de 39 millions de mètres cubes en 1960 à 29 millions en 1962, alors que le plan prévoyait pour cette dernière année une production de 40 millions de mètres cubes $\gg$.

Vingt-trois pour cent de la population mondiale se trouvent en Chine. Mais ce pays ne contient que 2,5 pour cent des forêts accessibles. Les ressources forestières sont donc minces par rapport à la population. « Même si l'on tient compte des plans actuels de reboisement, il est peu probable que les disponibilités locales pourront satisfaire les besoins futurs. L'ampleur des besoins de la Chine dissimule d'ailleurs ceux des autres pays de la région Asie/Pacifique. S'ils doivent être couverts par des importations, ce sera toute la situation commerciale actuelle qui en sera altérée 》, a dit M. Richardson.

«En Chine; la plus grande partie des forêts a été détruite au cours des siècles et il faut avoir vu l'étendue de l'érosion et la pénurie de produits forestiers qui en sont résultées. Partout, on constate cette pénurie. On utilise le bambou, la bagasse, la paille et l'herbe comme matériaux de construction. Le cinquième seulement des matériaux bruts qui entrent dans la fabrication du papier est de la pâte de bois. On utilise le béton ou l'acier au lieu de bois pour les couchettes dans les trains et l'on arrache par millions de jeunes plants d'arbres pour en faire du bois de feu $\gg$.

Selon l'étude, de nombreux centres de semences et quelque 30000 ha de pépinières ont été créés. Plus de 11000 étudiants ont été formés dans une vingtaine de collèges forestiers depuis 1952 et les Chinois espèrent former quelque 5000 spécialistes des eaux et forêts par an à partir de 1972 .

Trois grands problèmes se posent à la Chine dans le domaine de la foresterie, conclut M. Richardson. Ce sont: la plantation réussie de jeunes arbres sur des sites inhospitaliers, l'établissement d'un inventaire sylvicole et l'adoption de meilleures méthodes d'entretien des trois principales espèces d'arbres, et enfin l'amélioration de l'efficacité et du rendement des industries forestières existantes.

FAO.

\section{Eau et air dans les plans d'aménagement régionaux}

C'est sous ce thème général que se tiendra du $1^{\text {er }}$ au 4 mars 1965 dans les halles de la Foire Suisse d'Echantillons de Bâle, le $3^{\text {e }}$ Congrès scientifique PRO AQUA, organisé conjointement avec le Salon international PRO AQUA, eau, déchets, air. Comme ce fut le cas pour la manifestation précédente de 1962, le congrès - auquel participeront plus de 600 spécialistes d'Europe et d'outre-mer - sera présidé par le Professeur Otto Jaag, de Zurich.

Pour tous renseignements, prière de s'adresser au secrétariat du Salon PRO AQUA, case postale 4000, Bâle 21 (Suisse).

\section{Les poteaux en bois pour lignes de distribution d'énergie électrique}

En accord avec les services de l'Electricité de France, le Centre Technique du Bois vient de publier son cahier $\mathrm{n}^{\circ} 64$ consacré aux poteaux en bois destinés à supporter les lignes d'énergie électrique. Rédigé par M. LEPRINCE, Ingénieur à l'E.D.F., en collaboration avec le C.T.B., le cahier contient d'abord un rappel des caractères botaniques et des particularités anatomiques des essences utilisées pour les poteaux de ligne, puis l'auteur étudie successivement les altérations du bois naturel en service, les procédés d'imprégnation et les produits utilisés pour effectuer le traitement. 
Le bois offre, comme support de conducteurs électriques dans les lignes de transport d'énergie, des avantages certains et l'imprégnation permet de prolonger considérablement la durée de service des poteaux en bois.

On peut se procurer le cahier $\mathrm{n}^{\circ} 64$ au Centre Technique du Bois, 10, avenue de Saint-Mandé, Paris-12 ${ }^{\circ}$. Participation aux frais: 2,50 F. Envoi contre versement de $4 \mathrm{~F}$ au compte de chèques postaux C.T.B. Documentation, Paris 18108-93. Prière de mentionner sur le talon du virement: «Commande (à envoyer) d'un cahier 64 》. 